Horizons philosophiques

\title{
Esthétique de la répétition (Duchamp/Derrida)
}

\section{Rudy Steinmetz}

Volume 3, numéro 2, printemps 1993

Paysages esthétiques

URI : https://id.erudit.org/iderudit/800921ar

DOI : https://doi.org/10.7202/800921ar

Aller au sommaire du numéro

Éditeur(s)

Collège Édouard-Montpetit

ISSN

1181-9227 (imprimé)

1920-2954 (numérique)

Découvrir la revue

Citer cet article

Steinmetz, R. (1993). Esthétique de la répétition (Duchamp/Derrida). Horizons philosophiques, 3(2), 45-60. https://doi.org/10.7202/800921ar d'utilisation que vous pouvez consulter en ligne.

https://apropos.erudit.org/fr/usagers/politique-dutilisation/ 


\section{Esthétique de la répétition (Duchamp/Derrida)}

\section{L'art comme répétition}

L'introduction au Salon des Indépendants de New York, en 1917, du célèbre urinoir baptisé Fountain et signé R. Mutt, provoqua sans nul doute la percée la plus radicale des cloisons, jusque là maintenues étanches, entre le domaine des objets d'art et celui des objets ordinaires. Ce déplacement ou cette "décontextualisation" d'une vulgaire pissotière eut pour effet, non d'oblitérer le sens fonctionnel qu'on lui reconnaît habituellement, mais de lui adjoindre, de lui "greffer" un sens nouveau, celui — noble et élevé — dont s'entoure l'«aura» des productions dites "artistiques".

II ne saurait dès lors s'agir d'admettre, avec Marc Le Bot, que «la démarche de Marcel Duchamp, fabricant de readymade, consiste à arracher un objet au contexte dans lequel il a un sens utilitaire pour le transporter au musée où il perd ce sens ${ }^{1} "$. Si nous sommes d'accord avec la première partie de la proposition, la seconde en revanche n'est pas de nature à nous satisfaire. Qu'un objet soit bel et bien "arraché» à son lieu d'origine, nous le concèdons volontiers. Mais que, suite à cet arrachement, il perde le sens qui était le sien avant son extraction, nous ne voyons pas au nom de quoi Marc Le Bot est en droit de l'affirmer. II nous semble au contraire plus juste de dire que deux sens, l'un technique, l'autre esthétique, viennent à coexister dans un seul et même objet, produisant une sorte d'hybride. Sans cette tension plaçant l'objet à distance de luimême, provoquant en lui une "différence" insaisissable, le sentiment d'étrangeté qui secoue nos habitudes perceptives et provoque le malaise intellectuel ne serait pas éprouvé devant le

1. Marc Le Bot, “Marcel Duchamp et "ses célibataires, même" ", Esprit, n 79 (février 1992), p. 14. 
ready-made. De ce point de vue, Lévi-Strauss a raison de faire remarquer que, si, dans un premier temps, s'opère une «fission sémantique", une rupture du lien entre le signe et sa signification, celle-ci se réalise en définitive «au profit d'une fusion" ${ }^{2}$ qui conserve ce qu'elle a scindé. Ainsi se contracte, sur base de l'ancienne, «une nouvelle péréquation du rapport entre signifiant et signifié, une péréquation qui était dans le domaine du possible mais qui n'était pas ouvertement réalisée dans la situation primitive de l'objet ${ }^{3}$ ".

Avec l'entrée du ready-made dans l'espace muséal comme on dit aujourd'hui, c'est à une sorte de grande réserve de significations cachées derrière le voile de la classification des objets que l'on a accès tout d'un coup. Une espèce de paradigme sémantique du monde objectal se révèle soudainement dans la collusion syntagmatique d'un objet banal avec des objets réputés distingués. Par le biais de ce rapprochement singulier, via l'événement "corrupteur» ou "parasitaire» qui promeut le ready-made au rang d'objet d'art, se révèle la zone d'indécidabilité à partir de laquelle - mais aussi contre les ressources de laquelle - se spécifie tel ou tel domaine d'objets. Cette zone d'indécidabilité, il ne faudrait pas s'empresser de la réifier et y voir un système transcendantal des objets. Elle n'est en réalité rien d'autre que la possibilité qu'ils ont, en tant qu'artefacts, autrement dit signes matériels de l'activité humaine, d'être mis à l'écart de la sphère où ils fonctionnent et signifient habituellement pour s'intégrer ailleurs, dans un nouveau cadre contextuel au sein duquel ils recevront de nouvelles déterminations. Car les objets, si l'on y prend garde, ne sont assignés à une résidence précise que suivant l'usage de certaines conventions réprouvant toute vélléité de mixage ou de bâtardise. Le ready-made aussi bien que le ready-made réciproque ("se servir d'un Rembrandt comme table à repasser ${ }^{4}$ !») bouleversent cette logique dichotomique et font la

2. Georges Charbonnier, Entretiens avec Lévi-Strauss, Paris, UGE, 1961, p. 111.

3. Ibid., p. 112.

4. Marcel Duchamp, Duchamp du signe, Paris, Flammarion, 1975, p. 192. 
preuve que, en vertu d'une décision arbitrairement arrêtée de placer, ici ou là, la limite, chaque secteur d'objets est en réalité découpé sur un fond illimité de significations et d'usages potentiels qu'il est toujours possible de réactiver. C'est ce que semble indiquer l'adage postduchampien «tout est art», si l'on veut bien entendre par là que tout objet peut devenirune oeuvre d'art. Car il n'est pas nécessaire qu'il le devienne : il suffit qu'il le puisse en vertu d'une double révolution, de notre regard et de nos conceptions. Abstraction faite de tous les partages effectifs qui les répartissent et les organisent en un espace homogène, il faudrait pouvoir reconnaître, agissante en eux, cette possibilité de subversion des champs objectaux et, partant, qu'en matière d'art «il n'y a pas d'oeuvres, il n'y a qu'un effet d'oeuvre "».

À cette "esthétique du désoeuvrement ${ }^{6}$ " - qui est aussi une esthétique de la répétition - exploitée par Duchamp dans l'univers artistique et dans celui des objets ordinaires correspond, dans le champ plus vaste de la sémiologie telle qu'elle fut définie par Saussure, la déconstruction derridienne. En n'arrêtant pas de proclamer que la notion de «texte", qu'il exploite abondamment, n'exclut nullement la référence à la réalité extérieure, mais l'inclut au contraire ${ }^{7}$, Derrida vise à accéder aux conditions de possibilité de toute marque en général. Or, il semble bien que ce qui distingue le signe, n'importe quel signe - pas seulement l'oeuvre d'art ou l'objet usuel — soit sa

5. Bernard Pingaud, "L'objet littéraire comme "ready-made" $"$, L'Arc. Marcel Duchamp, Duponchelle, 1990, p. 20. Nous soulignons que Marcel Duchamp déclarait, à propos du "Grand Verre", qu'il s'agissait de proposer "la figuration d'un possible. [...] Le possible est seulement un umordant» physique [genre vitriol] brûlant toute esthétique ou callistiquen (Duchamp..., p. 104). A cet égard, Lyotard a parfaitement raison de faire valoir que, chez Duchamp, nous n'avons pas affaire à un monde d'objets fixes et donc de secteurs fixes d'activités déterminées, mais que tout est au contraire une question de transformations énergétiques, d'événements passagers et que, «quand il se passe quelque chose, le quelque chose ne se livre pas d'abord pour ce qu'il est, sans quoi il ne se passe rien" (Jean-François Lyotard, Les transformateurs Duchamp, Paris, Galilée, 1977, p. 114). L'oeuvre n'est donc rien d'autre qu'une concrétion momentanée, un instant de condensation entre des états instables, ce qui est appelé à passer.

6. Philippe Minguet, Sens et contresens de l'art, Bruxelles, De Boeck, 1992, p. 27-39.

7. Par exemple Limited Inc., Paris, Galilée, 1990, p. 253 et 273. 
capacité à être reproduit, répété, cité, c'est-à-dire, dans le cas le plus net, extrait de son contexte originaire et transféré vers un autre contexte où il pourra être repéré, identifié comme le "même» - dans un environnement (con)textuel différent. Pareille répétabilité peut ne pas affecter, de fait, une marque. II demeure que, étant une possibilité structurelle, elle empêchera toujours qu'un signe, fût-il non itéré, soit en coïncidence avec son sens. Dans son retentissant article sur Austin, Derrida désigne indifféremment cette "mobilité» sans contrainte du signe comme "citationnalité» ou «itérabilité générale $e^{8}$ ". C'est en elle et par elle que se déploie le règne d'une interprétation qui ne connaît pas de bornes : ce qui, en effet, assure la répétabilité et donc la lisibilité d'un signe est aussi ce qui met en péril cette lisibilité et ouvre la porte à la fiction. Cette fiction se réapplique sans cesse aux concepts - tels ceux d' «itérabilité» ou de «citationnalité» - qui voudraienten rendre compte etl'objectiver. II n'y a dès lors pas d'itérabilité pure, d'espace transcendantal de l'itérabilité, mais plutôt une itérabilité impure ou, comme l'amende souvent Derrida, une itérabilité «quasi» transcendantale, une itérabilité qui n'a de cesse de s'insinuer "entre le re- du répété et le re- du répétant, traversant et transformant la répétition ${ }^{9}$ ". Àl'instar du fameux Carré blanc sur fond blanc de Malévitch dont l'indiscernabilité entre le fond et la forme est patente, la répétabilité est à la fois l'espace transcendantal sur lequel s'enlève chaque répétition et l'empreinte laissée sur cet espace par celles-ci. II y a toujours retraversée de l'a priori par cela même que cet a priori paraît conditionner. La répétition est vouée à se répéter inlassablement. Ce qui explique pourquoi la déconstruction n'est pas, comme on le dit souvent, un travail de destruction, mais un travail sisyphéen de décomposition et de recomposition, des structures signifiantes en vue de promouvoir, par l'émergence de nouveaux corps textuels, l'insondable itérabilité qu'ils mettent en oeuvre.

8. II s'agit bien sûr de l'article «Signature événement contexte", repris dans Marges (Paris, Minuit, 1972, p. 365-393. Les citations figurent à la page 387).

9. Derrida, Limited..., p. 106. 
Le caractère illimité et somme toute machinal de cette "citationnalité" ou «itérabilité générale" conduit à penser qu'un signe peut toujours être libéré des intentions qui l'animent et coupé de toute "communication des consciences ${ }^{10}$ ". Cela revient à reconnaître qu'il ne possède pas d'identité absolue, qu'il est au contraire pourvu d' «une force de rupture avec [...] l'ensemble des présences qui organisent le moment de son inscription ${ }^{11}$ ". II ne sera donc plus possible de l'identifier d'un contexte à l'autre, à moins d'oublier qu'il est une sorte de catalyseur de significations qui diffractent ce que l'on pourrait encore percevoir, chez lui, comme un foyer de sens sous son apparente unité plastique. Mais, même celle-ci ne résiste pas à la turbulence répétitive. C'est qu'il n'y a pas d'atome insécable de l'expression et de la signification qui l'accompagne. Un mot peut être morcelé, divisé, émietté, et ses parties insérées ailleurs, dans un autre texte qui sera leur nouveau contexte, car la loi de répétabilité et de divisibilité des marques est plus fondamentale que les règles morphologiques qui, à un niveau plus régional et superficiel, régissent la configuration des vocables en chaque langage particulier.

\section{Signes sans site}

Ainsi que l'indique cette fois très justement Marc Le Bot dans un autre article, "avec l'entrée [...] de l'urinoir de Marcel Duchamp au Musée, la question de l'art ne porte plus du tout sur l'Art même. Mais sur ses bords, limites, marges ou margelles ${ }^{12}$,. Le processus de duplication auquel obéit le ready-made l'enveloppe d'une certaine duplicité : une "contamination" se produit entre l'objet fabriqué en grande série et la prétendue unicité de l'oeuvre d'art. Dès lors que, sans aucune transformation qualitative - ou presque - les objets les plus communs sont capables de se muer en objets dignes d'appréciation esthétique et figurer en bonne place parmi les chefs-d'oeuvre

10. Derrida, "Signature ...", p. 376.

11. Ibid,. p.377.

12. "Margelles du sens ou les musées de Marcel Duchamp", L'Arc..., p. 10. 
que nous a légués l'histoire de l'art, on ne sait plus ce qui fait la spécificité des uns et des autres. Le propre - s'il en est un du ready-made est de provoquer une espèce de "court-circuit culturel» aboutissant à un état de "double bind esthétique» de telle sorte que, s'il véhicule une contradiction, celle-ci consiste en ce que "le ready-made est et n'est pas un objet d'art ${ }^{13}$ ".

Comme l'indique lui-même Marcel Duchamp, le readymade "n'a rien d'unique ${ }^{14}$ ». C'est un objet essentiellement réplicable, appelé même à la réplication par ses vertus hautement mimétiques ${ }^{15}$. Le ready-made "aidé" élargit la sphère d'action de cette répétabilité et fait ressortir son caractère nonidentitaire. Cette légère correction apportée à un objet (par exemple en y appliquant une signature ou une phrase qui, disait Duchamp, vise «à emporter l'esprit du spectateur vers d'autres régions plus verbales $\left.{ }^{16} "\right)$, est une "transformation destinée à utiliser les petites énergies gaspillées ${ }^{17}$ ", et ce en vue de provoquer les plus surprenants effets de sens. On rapprochera ce labeur de récupération et de condensation énergétiques du travail auquel se livre la déconstruction et que Derrida, dans un entretien avec Lucette Finas, qualifiait de "travail économique : le plus grand rendement syntaxique et sémantique possible avec la moindre dépense $e^{18}$ ". Toute sa théorie de la "double écriture" ou de la "paléonymie" est contenue dans la brièveté de cette formule. Car qu'est-ce que l'écriture derridienne, sinon la reprise - "telle quelle" ou à peine "corrigée" - d'un mot ou d'un concept (par exemple, la "différance») qui, appartenant à un champ déjà constitué (dans ce cas-ci, le philosophique et le linguistique) et ne prenant sens qu'en lui, sert "de levier

13. Mathieu Kessler, "Le simulacre au Jeu de paume", Esprit..., p. 40.

14. Duchamp ..., p. 192.

15. Comme le dit Roger Dadoun, Duchamp c'est «l'Imitateur suprême", la "Mimésis absolue : le mime même d'un objet, c'est l'objet même" ("Rrose sschize", L'Arc..., p. 25).

16. Duchamp..., p. 192.

17. Ibid., p. 272.

18. Jacques Derrida, "Avoir l'oreille de la philosophie», entretien avec Lucette Finas, dans La quinzaine littéraire, $n^{\circ} 152$ (du 16 au 30 novembre 1972), p. 16. 
d'intervention [...] pour garder une prise sur l'organisation antérieure qu'il s'agit de transformer effectivement ${ }^{19}$ " . La double écriture ou paléonymie, on le voit, est le transfert, par la déconstruction, de l'opération duchampienne du ready-made.

Dépourvu donc de tout repère et critère pour répondre au besoin de savoir ce qui appartient ou n'appartient pas à la sphère artistique, nous sommes forcés de reconnaître que le monde de l'art est devenu, aujourd'hui beaucoup plus qu'auparavant, changeant et imprévisible. Tout objet, tout acte le produisant ou se produisant pour lui-même est à même de passer pour un chef-d'oeuvre. II n'y a plus de distinction marquée entre le "grand Art", c'est-à-dire ce qui longtemps fut pris pour l'Art tout court, et le non-art. Le problème est alors moins de savoir ce qu'est l'art puisque plus rien ne semble le différencier de son contraire que, comme y insiste Thierry de Duve, d'accepter "le pouvoir magique du mot "art"20». Par l'énonciation de ce simple vocable, l'institution artistique s'institue elle-même, d'elle-même, se confère, à partir de rien ou de n'importe quoi, une légitimité, se modifie et se transforme tout aussi bien selon ses propres décrets. II n'y a de l'art, ainsi que le soulignait déjà Arthur Danto ${ }^{21}$, que lorsque l'on dit qu'il y en a. Le mot "art» est un performatif et la réalité de l'art n'advient que par lui.

Ce que confirme exemplairement le ready-made Fountain qui dénonce et en même temps "récupère» l'arbitraire de la valeur artistique en érigeant en objet hautement culturel un ustensile destiné primitivement à ne satisfaire que des besoins biologiques. Plus encore qu'un objet - ce qu'il est assurément -il est un concept, "une phrase telle qu'elle s'épingle à un objet absolument quelconque, et qui dit : ceci est de l'art ${ }^{22}$ ". Incarna-

19. Jacques Derrida, Positions, Paris, Minuit, 1972, p. 96 . Nous soulignons le mot "transformer".

20. Thierry de Duve, Au Nom de l'art, Paris, Minuit, 1989, p. 17.

21. Voir "Le monde de l'art", traduit par Danielle Lories dans Philosophie analytique et esthétique, Paris, Méridiens Klincksieck, 1988, p. 183-198.

22. de Duve, Au Nom..., p. 113. 
tion emblématique du «nominalisme pictural ${ }^{23}$ ", le ready-made met la peinture en contradiction avec elle-même en survalorisant le conceptuel au détriment du perceptuel. En agissant aussi comme une prescription : celle qui enjoint à l'artiste «de faire n'importe quoi ${ }^{24}$ ” parce que la peinture et, d'une façon générale, l'art, c'est tout ce qu'il suffira de nommer "art" - par exemple «n'importe quoi» -, et ce au détriment des formes passées de l'art. Resterait évidemment à savoir ce que, depuis Duchamp, il est encore actuellement possible de "faire d'une liberté qui s'impose ou d'un ordre qu'il n'y a pas moyen d'enfreindre ${ }^{25}$ "? Question à laquelle nous ne pouvons ici apporter de réponse.

De la même manière que l'art affronte la question de ses frontières, ce qui intéresse Derrida dans la philosophie c'est qu'elle se pose comme un discours ayant pour objet d'établir et de "s'assurer la maîtrise de la limite ${ }^{26}$ ". Mais, à la différence de l'art contemporain qui la remet en cause et la problématise, la démarche philosophique classique a toujours visé à ce «que sa propre limite ne lui restât pas étrangère ${ }^{27}$ ", à garantir donc son autonomie. Bien que tendus apparemment vers des objectifs dissemblables (l'art moderne reprenant et commentant explicitement la tradition dont il est issu en vue de la fracturer; la philosophie cherchant, par la même opération, à en assurer l'indépendance), le résultat obtenu est similaire. C'est que, ainsi que s'en est avisé Derrida, les pratiques qui interrogent leurs propres marges sont, tôt ou tard, confrontées à l'ambiguilté de la notion de «limite»: est-ce qu'une limite n'est pas à la fois interne et externe à son champ d'application?

Ce que Derrida cherche d'abord à cerner dans la philosophie, de la même manière qu'ill'avait d'ailleurs fait pour le signe,

23. Thierry de Duve, Nominalisme pictural, Paris, Minuit, 1984. En particulier «Le ready-made et l'abstraction", p. 211-239.

24. de DUVE, Au Nom..., p. 119.

25. Ibid.

26. Derrida, Marges..., p. I.

27. Ibid. 
c'est une certaine généralité de son mode de fonctionnement. Selon lui, la pensée spéculative aurait pour but d'enrayer le pouvoir d'itérabilité des signes qui remet en question la participation d'un texte à un genre défini. Un des moyens qui procède à l'écartement d'une telle menace est l'élection de ce qu'il repère sous le nom de «signifié transcendantal». Cette instance représenterait la part intouchable et intouchée, inviolable et inviolée du discours philosophique, ce sur quoi il s'appuie et se fonde, qu'il peutéventuellement signifier mais qui reste inaltérée dans le procès même de son expression conceptuelle par quoi il vise à s'en approcher. Alors que les manières de dire varient, ce qu'il y a à dire ne varie pas. Ce ceest le point de convergence où se rassemblent toutes les perspectives, référence invariable qui expliquerait pourquoi la philosophie, quelle que soit sa configuration historique, est demeurée foncièrement fidèle au schéma du platonisme le plus conventionnel : s'approprier ce qui l'excède sans que cette part excessive ne souffre d'une désappropriation de soi.

Le même processus électif se serait donc déroulé, là dans le monde de l'art d'avant le ready-made qui vient le mettre en pièce, ici dans cette langue spécialisée qu'est la langue philosophique : ériger une pratique - artistique ou linguistique - en domaine spécialisé ayant son objet propre, sa technique propre et sa finalité propre; bref créer de toute pièce «la loi du genre». Mais cette élection- «érection" - comme aime à la sousentendre Derrida - ne va jamais sans défaillance. On l'a vu avec l'urinoir de Duchamp qui use et abuse de la fermeture fragile, parce que toute conventionnelle, du champ de la production artistique, champ dont les limites sont toujours décidées par des instances (critiques d'art, artistes, etc.) qui se disent compétentes et s'autorisent à légiférer en matière d'art. C'est ce conventionnalisme des critères que Derrida voudrait faire ressurgir sous l'apparente robustesse des constructions philosophiques. Et il le fait en montrant que, avant de renvoyer aux choses, les mots renvoient d'abord aux mots : pour ce qu'un réseau de "forces d'attraction cachées ${ }^{28}$ " ne cessent de

28. Jacques Derrida, La Dissémination, Paris, Seuil, 1972, p. 149. 
dissiper l'énergie sémantique canalisée dans la conceptualité philosophique et de la renvoyer vers tout "le système de la langue ${ }^{29}$ " sur lequel elle s'édifie. De là, chez Derrida, les fréquents recours aux définitions des dictionnaires qui sont autant de ready-made textuels réannexant la particularité du langage philosophique sur la généralité de sa matrice générative $^{30}$. Procédé dont la rigueur n'échappe pas à Meschonnic mais qui, cependant, continue de voir une «étrange logique" dans la "façon d'utiliser un dictionnaire et d'y rapporter la langue, au lieu de rapporter le dictionnaire à la langue ${ }^{31}$ ”. C'est que l'usage que l'on fait de la langue accapare des sens et en délaisse d'autres, virtuellement réactualisables, que les dictionnaires consignent au fur et à mesure du déroulement de I'histoire. Ils accumulent ainsi un nombre impressionnant de termes qui se font écho les uns les autres et dont il s'agira de réveiller les voix. Toute philosophie, quant à elle, opère une "sélection" en favorisant, à partir de la langue naturelle, la transition métaphorique, le "passage [...] d'un nonphilosophème dans un philosophème ${ }^{32}$ ». Sorte d'épuration par quoi des concepts adviennent et s'imposent au détriment des significations courantes qui les sous-tendent. "Le problème de la traduction" qui implique toujours, de l'avis de Derrida, une sélection, devient ainsi, et par excellence, le "problème du passage à la philosophie ${ }^{33}$ ». Chaque langage, suivant le degré

29. bid.

30. De même que la langue philosophique s'élabore à partir de la langue naturelle et selon une lente "usure" métaphorique, la peinture, aux dires de Duchamp, existe d'abord sous la forme d'un ensemble de couleurs que l'artiste choisit parmi ses tubes. Dès lors, nous pouvons en conclure que tous les textes philosophiques sont des ready-made usés et que "tous les tableaux du monde sont des Ready-mades aidés" (Marcel Duchamp, cité par Yves Arman, dans Marcel Duchamp joue et gagne, Marval, galerie Yves Arman, galerie Beaubourg, galerie Bonnier, 1984, p. 84).

31. Henri Meschonnic, "L'écriture de Derrida", Le Signe et le Poème, Paris, Gallimard, 1975, p. 412. II précise plus loin que cette référence très assidue aux articles du dictionnaire trouve son aboutissement dans le discours étymologique qui est «une prosopopée de la langue tenue par une pythie qui s'efface, transparente à son discours" (ibid., p. 481).

32. Derrida, La Dissémination..., p. 80.

33. Ibid. 
plus ou moins élevé de sa spécialisation terminologique, constitue de la sorte une «épure» du matériau linguistique de base à partir de quoi il s'enlève. De même que la prohibition de l'inceste promeut des alliances et en interdit certaines sur la base d'un fond biologique qui, a priori, n'impose ni n'empêche aucune relation interindividuelle mais, au contraire, les tolère toutes, de même les prohibitions discursives qui sont liées aux différents genres résorbent-elles la répétabilité différentielle et instaurent-elles, sur sa répression, des codes d'assemblage destinés à réguler les associations linguistiques. Le récit philosophique tisse donc sa trame au détriment de ramifications ignorées ou défendues. Ce sont ces ramifications de sens oubliées, écartées, voire même déniées - mais toujours prégnantes - que Derrida entend renouer aux fils du discours philosophique. Pour cet «homme-araignée», il n'y a pas de texte qui ne soit pris dans les mailles d'une texture plus large qui l'enveloppe, celle-ci «régénérant indéfiniment son propre tissu derrière la trace coupante, la décision de chaque lecture ${ }^{34}$ ».

La traduction d'une langue dans une autre ou d'une langue dans une même langue est une coupe chirurgicale dans la chair du langage. Sous la calme apparence du logos anesthésié se dissimule «un vivant sauvage, une animalité ambiguë ${ }^{35}$ » à laquelle Derrida veut redonner vigueur. Sorcier de l'avantgarde philosophique comme Duchamp était celui de l'avantgarde artistique, il prétend faire rejaillir de l'instrument stérilisé qu'est devenu le langage, tout son «pouvoir d'envoûtement, de fascination médusante, de transformation alchimique qui l'apparente à la sorcellerie et à la magie ${ }^{36}$ ", de la même façon que Duchamp, en exploitant la magie du mot «art», opérait, pour reprendre un titre connu, la «transformation du banal». Le langage est manifestement doué, pour Derrida, d'une force performative, il est un moyen d'action dont on a tort de réduire l'impact à la seule représentation neutre du réel : c'est, tout 
autant, un opérateur de transformation capable de modifier celui-ci. II faut - comme Duchamp l'avait fait pour la beauté, sorte de signifié transcendantal de l'esthétique - exorciser le leurre de la réalité, signifié transcendantal de la philosophie qui ampute le signifiant de sa puissance créatrice en donnant à croire que les mots sont de simples reflets d'un donné préexistant. C'est au sabbat des signes que ce diable d'homme nous convie, à la dissolution sacrilège des grandes oppositions qui structurent notre univers mental autant que matériel.

\section{La répétition comme art}

Qu'il faille ou que l'on puisse faire "n'importe quoi» en philosophie (et que la philosophie soit, peut-être, par contrecoup, n'importe quoi) est, tout comme dans le domaine des arts, une prise de position paradoxale en ce qu'elle repose sur l'a prioricité d'une structure itérative qui, comme on l'a vu, interdit à l'avance toute obligation ou toute interdiction de faire ou de ne pas faire. Nous serions depuis toujours condamnés à ne pas restreindre arbitrairement l'usage que nous faisons de nos signes. À ne pas établir de clivages génériques. C'est là notre devoir de liberté. II reste néanmoins qu'un choix dont la nature ne peut être déterminée à l'avance doit être posé pour ou contre le relâchement de la fonction symbolique. Car, de ce que le langage, étant originairement habité par la «différance», n'est investi d'aucune charge particulière et peut, de ce fait, sombrer dans une pure dépense gratuite et masturbatoire (Duchamp considérait la peinture comme une «masturbation olfactive»), il ne s'ensuit pas avec certitude que l'on retombe sans effort dans l'enfance des signes où prévaut le jeu des mots. Tout un cortège de discours adultes - philosophiques ou autres - ne cesse de défiler afin de nous en prémunir en faisant appel à notre sens du sérieux. L'infantilisation n'est pas une régression dont on serait victime, c'est une progression vers ce que Lyotard ne manquerait pas d'appeler notre «inhumanité» native, au sens où nous sommes toujours en manque ou en dette vis-à-vis de nous-mêmes, prématurés et qu'il nous faut respecter cette 
latence ${ }^{37}$. Il faut sans cesse se reconquérir, se rappeler à soimême, se répéter, revenir, toujours, à son origine perdue. Aucune de ces répétitions singulières n'épuise la répétabilité générale qui les conditionne. Mais il est vrai aussi que, à l'inverse, sans les premières, la seconde n'aurait aucune chance d'apparaître. C'est à ce jeu de la différance qui est aussi l'enfance de la pensée que, chacun à leur manière, Duchamp et Derrida se sont livrés.

Mais, si l'un est le «modèle» historique de l'autre n'est-ce pas que l'art ou la beauté $a$, depuis toujours, libéré exemplairement un tel jeu? En face de l'oeuvre qui, aux dires de Derrida, ne peut "être résumée en quelque simultanéité ou instantanéité absolues ${ }^{38}$ " selon les voeux structuralistes, qui participe d'une beauté dont le sens est incapable de se «laisser épouser ou épuiser par le géomètre ${ }^{39}$ ", n'éprouve-t-on pas, selon ce que Schiller, dans la lignée de Kant, concevait sous les espèces d'un «instinct de jeu", l'unité inaccessible, toujours "différée», "de la réalité et de la forme, du hasard et de la nécessité, de la passivité et de la liberté ${ }^{40}$ ". Si l'homme n'est pleinement et sérieusement homme "que là où il joue» et s'il «ne joue que là où [...] il est homme ${ }^{41}$ », c'est-à-dire lorsqu'il est confronté à la beauté qui concilie à la fois la plus grande indétermination matérielle et la plus stricte contrainte formelle, alors l'homme est en attente de son humanité. Car celle-ci ne peut se réaliser que dans la discorde de la force vitale et de la pensée rationnelle, dans le conflit que Schiller établissait entre l'«instinct sensible» et l'«instinct formel», dans l'«insondable [...] réciprocité d'action entre le fini et l'infini42 » sur quoi Derrida ouvre l'abîme de la différance.

37. Jean-François Lyotard, L'Inhumain, Paris, Galilée, 1988, p. 9-15.

38. L'Écriture..., p. 26.

39. Ibid., p. 35.

40. Friedrich von Schiller, Lettres sur l'éducation esthétique de l'homme, Paris, Aubier, Éditions Montaigne, 1943, quinzième lettre, p. 199.

41. Ibid., p. 205. Derrida, pour sa part, n'a eu de cesse de réaffirmer, comme en écho à Schiller, que "la question du «jeu» est [...] des plus sérieuses" (Du Droit à la philosophie, Paris, Galilée, 1990, p. 15).

42. Ibid., p. 199. 
Certes l'unité restait promise pour ce disciple de Kant qu'était Schiller; elle est par avance démentie aux yeux de Derrida. Ici et là la différance s'exprime différemment, la répétition se répète avec quelques nuances. Quoiqu'il en soit de l'issue ou du départ, la même tension subsiste entre le fini et l'infini. Cette tension qui ne peut trouver sa juste expression est ce qui se répète indéfiniment, selon des modalités diverses et sans que la somme en soit totalisable. Cela est très sérieux et très amusant-comme une pissotière ou une roue de byciclette dans un musée. De se prêter à ce petit jeu prête aussi à rire et à pleurer, les deux à la fois tant il est vrai que "le comique absolu, c'est l'angoisse devant la dépense à fonds perdus, devant le sacrifice absolu du sens ${ }^{43}$ ". Ce mouvement répétitif inlassable qui affecte nos symboles et nos signes n'est pas luimême symbolique ou significatif. Et il serait risible ou trop sérieux de vouloir qu'il en soit autrement. La répétition ne se théorise pas, elle se pratique. Elle est un art à l'égal de cette écriture que Theuth remit en cadeau au roi ${ }^{44}$ et dont Derrida se fit l'artiste. Art à l'oeuvre dans tous les arts - de la parole ou plastiques - elle est comme "cette imagination qui aux yeux de Kant était déjà en elle-même un "art", était l'art lui-même qui originairement ne distingue pas entre le vrai et le beau ${ }^{45}$ ". Or cet art, qu'on s'en ressouvienne, demeurait caché dans les profondeurs de l'âme, se soustrayant à l'entendement et ne se donnant à voir qu'au travers des réalisations de l'imagination livrée à elle-même. C'est pourquoi, avec la répétition telle que la conçoit Derrida, advient l'impérieuse "nécessité du spectacle ${ }^{46}$ ". C'est pourquoi aussi tout est, en quelque façon et à commencer par la répétition, "oeuvre" chez lui, car on ne peut produire ou "décrire que l'oeuvre et le fait de la différance, les différences déterminées et les présences déterminées auxquelles elle donne lieu ${ }^{47}$ ". Parce que la répétition, en tant que

43. L'Écriture..., p. 377.

44. La Dissémination..., p. 84-85.

45. Derrida, L'Écriture..., p. 16.

46. bid., p. 379.

47. Jacques Derrida, De la Grammatologie, Paris, Minuit, 1967, p. 92. 
telle, nouménalement, échappe à toute forme de présentification, on n'échappe pas à l'ordre du déplacement phénoménal et à une certaine "esthétisation de la répétition", si l'esthétique a justement affaire, comme l'indique son étymologie, avec ce qui se phénoménalise.

Que la répétabilité soit la condition de possibilité de l'art (Duchamp), et plus généralement du signe (Derrida), que cette répétabilité soit par ailleurs elle-même un art, autrement dit l'art de l'art, c'est ce que Philippe Minguet a su très justement faire valoir en soulignant, un peu contre la thèse de Benjamin, que «l'art [...] existe à partir du moment où l'on admet la répétition de ses produits ${ }^{48}$ ". II reviendrait à la pratique moderne de l'art — et à Duchamp en particulier — d'avoir mis à jour le principe essentiel de toute activité artistique en administrant concrètement la preuve que «la reproduction assure le passage de la valeur fonctionnelle à la valeur spectaculaire, ou plutôt [...] réduit à elle-même la valeur d'art originellement impliquée $e^{49}$ ". L'art, en son unité, ne trouverait à se réaliser que dans la répétition infinie de ses oeuvres. II ne serait ainsi lui-même et appelé à lui-même qu'au moment où le risque est le plus grand de le voir disparaître dans une activité ordinaire en laquelle on ne l'avait jamais reconnu et dans laquelle son mystère se reploie. Comme le dit Derrida, "si le beau ne perd rien à être reproduit [...], c'est que dans la "première fois" de sa reproduction, il était déjà essence reproductive ${ }^{50}$ ". II n'y aurait donc pas antinomie entre l' "unicité» et la «multiplicabilité» de l'oeuvre d'art : la première trouverait au contraire dans la seconde le ressort même de sa "définition $^{51}$ » - pour reprendre une expression de Gianni Vattimo désignant le fait que l'art est toujours en quête de sa propre nature plutôt que détenteur de celle-ci.

48. Philippe Minguet, «Unicité et multiplicabilité en art (le statut esthétique de la reproduction)", Sens..., p. 49.

49. Ibid.

50. Derrida, De la Grammatologie..., p. 196.

51. Minguet, Sens..., préface, p. IX. La thèse de Philippe Minguetn'est pas absolument en contradiction avec celle défendue par Benjamin dans son célèbre article "L'oeuvre d'art à l'ère de la reproductibilité technique" (dans Poésie et révolution, 
L'histoire de l'art et celle de la philosophie ne seraient donc que des reprises perpétuelles et parallèles du principe de répétabilité. Mais avec Duchamp et Derrida on arrive à un point d'exacerbation de ce principe, à un point de croisement où l'art ne peut plus se passer d'une approche philosophique qui le problématise, et la philosophie d'une pratique artistique qui la mette au travail jusqu'à la rendre impossible. Le ready-made, on l'a dit tout à l'heure, est plus une réflexion, un commentaire qui incite à la reconsidération de la tradition artistique qu'un "objet" d'art, la "chose" artistique étant précisément, comme dirait Heidegger, ce qui, avec lui, fait question. De son côté, la pensée déconstructrice apparaît bien plutôt comme une espèce de représentation - au sens théâtral du terme - de mise en oeuvre artistique de la différance et de la répétition que comme leur explicitation (que l'on songe, par exemple, aux "collages" derridiens de "Tympan" ou de Glas). Il y a là, de part et d'autre, une incitation à mélanger les genres, à franchir les barrières qui séparaient encore, il y a peu, la théorie et la pratique. De l'art comme philosophie à la philosophie comme art, il y a le déploiement d'une circularité que la répétition génère autant qu'elle est générée par eux.

\section{Rudy Steinmetz Université de Liège, Belgique}

\footnotetext{
traduit de l'allemand et préfacé par Maurice de Gandillac, Denoël, 1971, p. 171210). Ce qu'affirme Minguet c'est que la reproduction esthétique - qu'il ne confond pas avec la reproduction économique - tend à l'infini vers l'unité de l'art, sans que celle-ci soit le moins du monde remise en cause par le fait qu'elle soit reproductible. Ce que Benjamin appelait l' «aura" de l'oeuvre d'art et que, très proche en cela de Heidegger, il définissait comme «l'unique apparition d'un lointain" (ibid., p. 178.), est chez Minguet ce qui ne cesse d'aimanter, dans son recul inappropriable, la répétition. Mais là où nos deux auteurs divergent, c'est dans le rôle qu'ils confèrent chacun à cette dernière. Alors que pour Benjamin, comme on le sait, la répétition altère l'aura de l'oeuvre, pour Minguet, au contraire elle la promeut. Encore qu'une note du premier ne soit pas si éloignée que ça de la pensée du second. Lorsqu'il annonce que "l'authenticité échappeà toute reproduction" et qu'il ajoute directement après «que le développement intensif de certains procédés techniques de reproduction a permis d'établir des différenciations et des degrés dans l'authenticité ellemême" (ibid., p. 175, note 1), Benjamin ne laisse-t-il pas entendre que la reproduction conditionne, dans une certaine mesure, l'authenticité du produit artistique puisqu'elle permet d'y établir une gradation?
} 